Pacific Journal of Mathematic 


\title{
A CONTINUOUS PARTIAL ORDER FOR PEANO CONTINUA
}

\author{
VIRGINIA KNIGHT
}

\begin{abstract}
A theorem of $R$. J. Koch states that a compact continuously partially ordered space with some natural conditions on the partial order is arcwise connected. L. E. Ward, Jr., has conjectured that Koch's arc theorem implies the well-known theorem of R. L. Moore that a Peano continuum is arcwise connected. In this paper Ward's conjecture is proved.
\end{abstract}

1. Preliminaries. If $\Gamma$ is a partial order on a set $X$ we will write $x \leqq_{\Gamma} y$ or $x \leqq y$ for $(x, y) \in \Gamma$. We will let $L(a)=\{x:(x, a) \in \Gamma\}$. If $X$ is a topological space, then $\Gamma$ is a continuous partial order on $X$ provided the graph of $\Gamma$ is closed in $X \times X$. If $\Gamma$ is a continuous partial order on the space $X$, then $L(x)$ is a closed set for every $x \in X$. A zero of a continuously partially ordered space $X$ is an element 0 such that $0 \in L(x)$ for all $x \in X$. An arc is a locally connected continuum with exactly two noncutpoints. A real arc is a separable arc. A Peano continuum is a locally connected metric continuum.

We will use the following statement of Koch's arc theorem.

THEOREM 1. If $X$ is a compact continuously partially ordered space with zero such that $L(x)$ is connected for each $x \in X$, then $X$ is arcwise connected.

We will show that Peano continua admit such partial orders by proving the following:

THEOREM 2. If $X$ is a compact connected locally connected metric space, then $X$ admits a continuous partial order with a zero such that $L(x)$ is connected for all $x \in X$.

The proof of this theorem will use some definitions and results due to R. H. Bing [1]. An $\varepsilon$-partition $\mathscr{P}_{\varepsilon}$ of a subspace $K$ of a metric space $M$ is a finite set of closed subsets of $M$, each with diameter less than $\varepsilon$, the union of which is $K$, and such that the interiors in $M$ of all the elements of $\mathscr{P}_{\varepsilon}$ are nonempty, connected, dense in the closed subset, and are pairwise disjoint. The subspace $K$ is partitionable if for each positive number $\varepsilon$, there exists an $\varepsilon$-partition of $K$.

Lemma 1. Let $M$ be a compact connected locally connected metric space. For each positive number $\varepsilon$ there exists an $\varepsilon$-partition $\mathscr{P}_{\varepsilon}$ of 
$M$ such that each element of $\mathscr{P}_{\varepsilon}$ is partitionable.

Bing proves this lemma in [1].

The proof of the Theorem 2 will follow in two parts. In the first part a relation $\Delta$ will be constructed on the Peano continuum $X$. The second part will be concerned with proving that $\Delta$ is the desired partial order on $X$. We will let $d$ denote the metric on $X$.

2. The construction of the relation $\Delta$. We will define inductively a sequence $\{\mathscr{F}(i)\}_{i=1}^{\infty}$ of finite partitions of $X$. With each partition we will associate a relation $\delta_{i}$. The set $\left\{\delta_{i}\right\}_{i=1}^{\infty}$ will be a nest of closed subsets of $X \times X$ and $\Delta=\cap \delta_{i}$ will be the desired partial order on $X$.

First choose an arbitrary element of $X$. Call this element 0 . This will be the 0 of the partial order to be constructed on $X$.

We will now construct the relation $\delta_{1}$ as the first step of the induction.

Let $\mathscr{F}(1)$ be a finite partition on $X$ such that for $F \in \mathscr{F}(1)$, $\operatorname{diam}(F)<1 / 2$, and such that $F$ is partitionable. We will classify the elements of $\mathscr{F}(1)$ according to how "far away" they are from 0. Let $\mathscr{F}(1,0)$ be the set $\{F \in \mathscr{F}(1): 0 \in F\}$. If $\mathscr{F}(1, i)$ has been defined for $i=1,2, \cdots, t-1$, let

$$
\mathscr{F}(1, t)=\left\{F \in \mathscr{F}(1)-\bigcup_{i=0}^{t-1} \mathscr{F}(1, i): F \cap(\cup \mathscr{F}(1, t-1)) \neq \varnothing\right\} .
$$

If $F$ is an element of $\mathscr{F}(1, t)$ we will say $F$ has order $t$. Because $\mathscr{F}(1)$ is a cover of the connected set $X$ with connected sets, there is a chain of elements of $\mathscr{F}(1)$ between any two points of $X$. That is, if $F$ is an element of $\mathscr{F}(1)$ then there exists some integer $t$ and a set $\left\{F_{i}\right\}_{i=0}^{t} \subset \mathscr{F}(1)$ such that $0 \in F_{0}, F=F_{t}$ and for $i, j \in\{0,1, \cdots, t\}$ $F_{i} \cap F_{j} \neq \varnothing$ if and only if $|i-j| \leqq 1$. This is the condition necessary for $F$ to have order $t$. Thus order is defined for all elements of $\mathscr{F}(1)$.

We now define sets $J(F)$, for $F \in \mathscr{F}(1)$, which will be in a sense "predecessors" of the elements of $F$. For $F \in \mathscr{F}(1,0)$ let $J(F)=F$. If $J(F)$ has been defined for $F \in \mathscr{F}(1, t-1)$ and if $F_{t} \in \mathscr{F}(1, t)$ let

$$
J\left(F_{t}\right)=F_{t} \cup \cup\left\{J(F): F \cap F_{t} \neq \varnothing, F \in \mathscr{F}(1, t-1)\right\} .
$$

We now define the relation $\delta_{1}$ on $X$ by defining for all $x \in X$ the set $L_{1}(x)=\left\{y:(y, x) \in \delta_{1}\right\}$. Set

$$
L_{1}(x)=\cup\{J(F): x \in F \in \mathscr{F}(1)\} .
$$

The relation $\delta_{1}$ is reflexive but not anti-symmetric or transitive. 
In order to define the relations $\delta_{2}, \cdots, \delta_{n}$, it will be useful to introduce some additional notation. Let $F$ be an arbitrary fixed element of $\mathscr{F}(1, t)$ for some nonnegative integer $t$. Let $\partial F$ denote the boundary of $F$. For $t=0$, let $\mathscr{E}_{*}(F)=\{0\}$, and for $t>0$, let

$$
\mathscr{E}_{*}(F)=\{E \in \mathscr{F}(1, t-1): E \cap F \neq \varnothing\} .
$$

Notice that $\mathscr{E}_{*}(F)$ is not empty by (1) since $F \in \mathscr{F}(1, t)$. Let

$$
\partial_{*} F=F \cap\left[\cup \mathscr{E}_{*}(F)\right] \text {. }
$$

Except for the case when $t=0$ and $\partial_{*} F=\{0\}, \partial_{*} F$ is that part of the boundary of $F$ which is also part of the boundary of sets of order $t-1$. Let

$$
\mathscr{E}(F)=\left\{E \in \mathscr{F}(1): E \neq F \text { and } E \cap F-\partial_{*} F \neq \varnothing\right\} .
$$

That is, $\mathscr{E}(F)$ is the set of elements of $\mathscr{F}(1)$, other than $F$ itself and the sets of order $t-1$, whose intersection with $F-\partial_{*} F$ is not empty. Note that the elements of $\mathscr{E}(F)$ either have order $t$ or order $t+1$. Let $\mathscr{E}^{*}(F)$ be the set $\left\{E \in \mathscr{E}(F): F \in \mathscr{E}_{*}(E)\right\}$ and let

$$
\partial^{*} F=\cup\{F \cap E: E \in \mathscr{E} *(F)\} .
$$

Then $\mathscr{E}^{*}(F)$ is the set of sets in $\mathscr{F}(1)$ which have order $t+1$ and have a nonempty intersection with $F$. The sets $\mathscr{E}(F)$ and $\mathscr{E}^{*}(F)$ may be empty. For $E \in \mathscr{E}(F) \cup \mathscr{E}^{*}(F)$ let $\partial_{E}(F)=E \cap F$.

If $\mathscr{E}^{*}(F)$ is not empty, let $\rho(F)$ be $d\left(\partial_{*} F, \partial^{*} F\right)$. Thus $\rho(F)$ is the infimum of the distances between the points of $F$ which are also in the sets of order $t-1$ and those points of $F$ which are also in sets of order $t+1$. This distance is positive since, by (1), for each $E \in \mathscr{E}^{*}(F), \partial_{E} F$ and $\partial_{*} F$ are disjoint closed sets. If $\mathscr{E}^{*}(F)$ is empty, let $\rho(F)$ be $\operatorname{diam}(F)$.

The remainder of the construction of $\delta_{2}$ generalizes directly to the construction of $\delta_{n}$. Thus we will assume that $\mathscr{F}(n)$, a partition of $X$, and the sets $\mathscr{F}(n, t)$ have been defined for $t=0,1, \cdots$, and that for $F \in \mathscr{F}(n), \partial_{*} F, \mathscr{E}_{*}(F), \mathscr{E}(F), \mathscr{E}^{*}(F), \partial^{*} F$ and $\rho(F)$ have been defined and that for each $E \in \mathscr{E}(F) \cup \mathscr{E}^{*}(F), \partial_{E} F$ has been defined. We will now define some special subsets of each $F \in \mathscr{F}(n)$ which we will use to define the relation $\delta_{n}$.

In order for the final relation $\Delta$ to be transitive it will be necessary that the elements of $\partial F-\left(\partial_{*} F \cup \partial^{*} F\right)$ have no successors in the relation $\Delta$. To this end we want to find for each $E \in \mathscr{E}(F) \cup \mathscr{E}^{*}(F)$ a partitionable subset of $F$ which contains $\partial_{*} F$ and $\partial_{E} F$ but contains no points of $\partial F$ which are not "close" to $\partial_{*} F$ or $\partial_{E} F$. We use the following lemma. 
LEMMA 2. Let $\varepsilon>0$ and let $F$ be a partitionable compact subset of a metric space $X$ such that the interior of $F$ is connected and locally connected. Let $B_{0}$ be either a nonempty closed subset of $\partial F$ or a point in the interior of $F$. Let $\left\{B_{i}\right\}_{i=0}^{m}$ be a finite set of nonempty closed subsets of $\partial F$, such that $\bigcup_{i=0}^{m} B_{i} \supset \partial F$. Then there exists $a$ set $\left\{C_{i}\right\}_{i=0}^{m}$ of partitionable subsets of $F$ such that for $i=0,1, \cdots, m$ $C_{i}$ is closed, (int $\left.C_{i}\right) \cup B_{i} \cup B_{0}$ is connected, $B_{i} \subset C_{i}$ and if $x \in \partial F \cap C_{i}$ then either $d\left(x, B_{i}\right)<\varepsilon$ or $d\left(x, B_{0}\right)<\varepsilon$. Further $C_{0} \subset C_{i}, i=0,1, \cdots, m$ and $F=\bigcup_{i=0}^{m} C_{i}$.

Proof. By Lemma $1, F$ is partitionable so let $\mathscr{P}(F)$ be a partition of $F$ such that for $P \in \mathscr{P}(F)$, diam $(P)<\varepsilon / 2$ and $P$ is partitionable.

For $x \in$ int $F$ let $U_{x}$ be a connected open set containing $x$ whose closure misses $\partial F$. Let $\mathscr{C}=\left\{U_{x}: x \in \operatorname{int} F\right\}$. For each $P \in \mathscr{P}(F)$ choose $x(P) \in \operatorname{int} P \cap \operatorname{int} F$ and let

$$
Q=\{x(P): P \in \mathscr{P}(F)\} \cup \cup\{P \in \mathscr{P}(F): P \cap \partial F=\varnothing\} .
$$

Let $\mathscr{U}_{1}$ be a finite cover of the closed set $Q$ by elements of $\mathscr{U}$. We can write $\mathscr{U}_{1}=\left\{U_{i}\right\}_{i=1}^{k}$. Now fix some element $P_{0} \in \mathscr{P}(F)$ such that $P_{0} \cap B_{0} \neq \varnothing$. The interior of $F$ is connected by the connected open sets of $\mathscr{U}$, so that for each $U_{i} \in \mathscr{U}_{1}$ there exists $\left\{U_{i j}\right\}_{j=0}^{k(i)} \subset \mathscr{U}$ such that $x\left(P_{0}\right) \in U_{i 0}, U_{i k(i)}=U_{i}$ and $U_{i j} \cap U_{i l} \neq \varnothing$ if and only if $|j-l| \leqq 1$. That is, there is a finite chain of sets of $\mathscr{C}$ connecting each element of $\mathscr{U}_{1}$ with $x\left(P_{0}\right)$. Let

$$
\begin{aligned}
\mathscr{C}^{\prime}=\left\{U_{i j}: i=0\right. & , \cdots, k ; j=0, \cdots, k(i)\} \\
& \cup\left\{P \in \mathscr{P}(F): P \cap B_{0} \neq \varnothing\right\} .
\end{aligned}
$$

Note that $\cup \mathscr{U}^{\prime}$ is a connected subset of $F$ and that if $x \in C l\left(\cup \mathscr{C}^{\prime}\right)$ and $d\left(x, B_{0}\right)>\varepsilon / 2$, then $x \notin \partial F$. This is because the boundary of each element of $\mathscr{C}$ misses the boundary of $F$, so that if $x$ were in $\partial F, x$ would be an element of $P$ for some $P \in \mathscr{P}(F)$ such that $P \cap B_{0} \neq \varnothing$ and we have that $\operatorname{diam}(P)<\varepsilon / 2$. Also note that

$$
F \subset\left(\cup \mathscr{U}^{\prime}\right) \cup \cup\{P \in \mathscr{P}(F): P \cap \partial F \neq \varnothing\}
$$

since $\mathscr{W}_{1} \subset \mathscr{U}^{\prime}$ and $\mathscr{U}_{1}$ is a cover of $\cup\{P \in \mathscr{P}(F): P \cap \partial F=\varnothing\}$.

Now consider $\mathscr{U}_{2}=\left\{U \in \mathscr{U}^{\prime}: \bar{U} \cap \partial F=\varnothing\right\}$. Let

$$
\nu(F)=\min \left\{\varepsilon / 2, \min \left\{d(\bar{U}, \partial F): U \in \mathscr{U}_{2}\right\}\right\} .
$$

For each $P \in \mathscr{P}(F)$ let $\mathscr{C}(F, P)$ be a partition of $P$ such that if

$$
F^{\prime} \in \mathscr{G}(F, P), \text { then } \operatorname{diam}\left(F^{\prime}\right)<\frac{\nu(F)}{4}
$$

and $F^{\prime}$ is partitionable. Let 


$$
\mathscr{C}(F)=\cup\{\mathscr{P}(F, P): P \in \mathscr{P}(F)\} .
$$

We are ready now to define the sets $C_{i}, i=0,1, \cdots, m$. The set $C_{0}$ will meet $\partial F$ only "close" to $B_{0}$ and $C_{i}, i=1, \cdots, m$ will meet $\partial F$ only "close" to $B_{i}$ or $B_{0}$. Let $D=\left[\left(\cup \mathscr{C}^{\prime}\right)-\partial F\right] \cup B_{0}$. The set $D$ is a connected subset of (int $F) \cup B_{0}$. Let

$$
C_{0}=\cup\left\{F^{\prime} \in \mathscr{C}(F): F^{\prime} \cap D \neq \varnothing\right\} \text {. }
$$

Because $D$ is connected and covered by $\mathscr{G}(F), C_{0}$ is a closed and connected subset of $F$. Also, if $x \in C_{0} \cap \partial F$, then $d\left(x, B_{0}\right)<\varepsilon$, for if $x \in C_{0}-B_{0}$ then $x \in F^{\prime} \in \mathscr{C}(F)$ such that $F^{\prime} \cap D \neq \varnothing$. Consequently there exists a $U \in \mathscr{U}^{\prime}$ such that $F^{\prime} \cap U \neq \varnothing$. It then follows that if $x$ were in $F^{\prime} \cap \partial F$ then, by definition of $\nu(F), U=P$ for some $P \in \mathscr{P}(F)$ such that $P \cap B_{0} \neq \varnothing$, and

$$
d\left(x, B_{0}\right) \leqq \operatorname{diam}\left(F^{\prime}\right)+\operatorname{diam}(P)<\frac{\nu(F)}{4}+\varepsilon / 2 \leqq \varepsilon / 8+\varepsilon / 2<\varepsilon .
$$

If we let $C_{0}^{\prime}=\left[(\operatorname{int} F) \cap C_{0}\right] \cup B_{0}$, then $C_{0}^{\prime}$ is connected because $C_{0}^{\prime}$ contains $D$ and

$$
C_{0}^{\prime}=\cup\left\{\left[F^{\prime} \cap(\operatorname{int} F)\right] \cup\left[F^{\prime} \cap B_{0}\right]: F^{\prime} \subset C_{0}\right\},
$$

which is a union of connected sets which cover $D$ and each of which has nonempty intersection with $D$.

Now let

$$
C_{i}=C_{0} \cup \cup\left\{\cup \mathscr{C}(F, P): P \in \mathscr{P}(F), P \cap B_{i} \neq \varnothing\right\} .
$$

We see that $C_{i}$ is a closed subset of $F$ and it is connected because $C_{0}$ and each $P \in \mathscr{P}(F)$ is connected and $x(P) \in P \cap C_{0}$. Let $C_{i}^{\prime}=$ [(int $F) \cap C_{i}$ ] $\cup B_{i} \cup B_{0}$. Then $C_{i}^{\prime}$ is a connected subset of $F$, for

$$
C_{i}^{\prime}=C_{0}^{\prime} \cup \cup\left\{[P \cap \operatorname{int} F] \cup\left[B_{i} \cap P\right]: P \in \mathscr{P}(F), P \cap B_{i} \neq \varnothing\right\},
$$

and $C_{0}^{\prime}$ and $[P \cap \operatorname{int} F] \cup\left[P \cap B_{i}\right]$ are connected and $x(P) \in C_{0}^{\prime} \cap P \cap \operatorname{int} F$ for each $P \in \mathscr{P}(F)$.

Further note that if $x \in C_{i} \cap \partial F$, then either $d\left(x, B_{i}\right)<\varepsilon$ or $d\left(x, B_{0}\right)<\varepsilon$. Also $F$ is a subset of $\bigcup_{i=0}^{m} C_{i}$.

This completes the proof of Lemma 2 .

To apply this lemma to the theorem we let $\varepsilon=\rho(F) / 3, B_{0}=\partial_{*} F$ and $\left\{B_{i}\right\}_{i=1}^{m(F)}=\left\{\partial_{E} F: E \in \mathscr{E}(F) \cup \mathscr{E}^{*}(F)\right\}$. Thus for $F \in \mathscr{F}(n)$ we get sets $C_{i}, i=0,1, \cdots, m(F)$ satisfying the conditions of the lemma. For clarity we will sometimes write $C(F)$ for $C_{0}(F)$ and use $C(F, E)$ for $C_{i}(F)$ when $B_{i}=\partial_{E} F$ for $E \in \mathscr{E}(F) \cup \mathscr{E}^{*}(F)$. We will also use $C^{\prime}(F)$ for $C_{0}^{\prime}$ and $C^{\prime}(F, E)$ for $C_{i}^{\prime}$.

We will now define the relation $\delta_{n}$ on $X$. First we inductively 
define sets $J(F)$ and $J(F, E)$ for each $F \in \mathscr{F}(n), E \in \mathscr{E}(F) \cup \mathscr{E}^{*}(F)$. The elements of $J(F)$ and $J(F, E)$ will, in a sense, be "predecessors" of the elements of $C(F)$ and $C(F, E)$ respectively.

For $F \in \mathscr{F}(n, 0)$, let $J(F)=C(F)$ and for $E \in \mathscr{E}(F) \cup \mathscr{E}^{*}(F)$ let $J(F, E)=C(F, E) \cup J(F)$. Then suppose $J(F)$ and $J(F, E)$ have been defined for all $F \in \mathscr{F}(n, t-1), E \in \mathscr{E}(F) \cup \mathscr{E}^{*}(F)$. Let $F$ be an element of $\mathscr{F}(n, t)$. Define

$$
\begin{aligned}
& J(F)=C(F) \cup\left\{J\left(F_{*}, F\right): F_{*} \in \mathscr{E}_{*}(F)\right\} \text { and let } \\
& J(F, E)=C(F, E) \cup J(F) \text { for } E \in \mathscr{E}(F) \cup \mathscr{E}^{*}(F) .
\end{aligned}
$$

Thus we can define $J(F)$ and $J(F, E)$ for all

$$
F \in \mathscr{F}(n), E \in \mathscr{E}(F) \cup \mathscr{E}^{*}(F) .
$$

The sets $J(F)$ and $J(F, E)$ are each closed since they are a finite union of closed sets. Also $J(F, E)$ is connected if $J(F)$ is connected since for each $E \in \mathscr{E}(F) \cup \mathscr{E}^{*}(F), C(F, E)$ contains $C(F)$. But $J(F)$ is connected since if $F_{*} \in \mathscr{E}_{*}(F)$ then for each $P \in \mathscr{P}\left(F_{*}\right)$ such that

$$
P \cap F \neq \varnothing, P \cap \partial_{*} F \cap C\left(F_{*}, F\right) \neq \varnothing .
$$

Thus $C(F)$ is not separated from $C\left(F_{*}, F\right)$ for any $F_{*} \in \mathscr{E}_{*}(F)$.

We will let $L_{n}(x)=\left\{y:(y, x) \in \delta_{n}\right\}$ and define $\delta_{n}$ by defining the sets $L_{n}(x)$ for all $x \in X$. Let $x \in X$ and $F \in \mathscr{F}(n)$. If $x \notin F$, let $K_{F}(x)=\varnothing$. If $x \in F$ and $x \in C(F)$, let $K_{F}(x)=J(F)$. If $x \in F$ and

$$
x \in \cup\left\{C(F, E): E \in \mathscr{E}(F) \cup \mathscr{E}^{*}(F)\right\}-C(F)
$$

there exists some $E \in \mathscr{E}(F) \cup \mathscr{E}^{*}(F)$ such that $x \in C(F, E)$, so let

$$
K_{F}(x)=\cup\left\{J(F, E): x \in C(F, E), E \in \mathscr{E}(F) \cup \mathscr{E}^{*}(F)\right\} .
$$

Then let

$$
L_{n}(x)=\cup\left\{K_{F}(x): F \in \mathscr{F}(n)\right\} .
$$

Then $L_{n}(x)$ is closed and connected for each $x$, for it is a nonempty finite union of closed sets, and the nonempty sets comprising that union are each connected and contain $x$.

The relation $\delta_{n}$ is closed because

$$
\begin{aligned}
\delta_{n}= & \cup\{C(F) \times C(F): F \in \mathscr{F}(n)\} \\
& \cup \cup\left\{C\left(F^{\prime}, E^{\prime}\right) \times C(F, E): F \in \mathscr{F}(n),\right. \\
& \left.E \in \mathscr{E}(F) \cup \mathscr{E}^{*}(F), C\left(F^{\prime}, E^{\prime}\right) \subset J(F, E)\right\}
\end{aligned}
$$

which is a finite union of products of closed sets.

To complete the induction we will assume $\delta_{n}$ has been defined and we will define the sets, $\mathscr{F}(n+1), \mathscr{F}(n+1, t), t=0,1, \cdots$, and for 
each $F \in \mathscr{F}(n+1)$ we must define $\partial_{*} F, \mathscr{E}_{*}(F), \mathscr{E}(F), \mathscr{E}^{*}(F), \partial^{*} F$, $\rho(F)$ and for each $E \in \mathscr{E}(F) \cup \mathscr{C}^{*}(F), \partial_{E} F$.

First let $\mathscr{F}(n+1)=\bigcup\{\mathscr{C}(F): F \in \mathscr{F}(n)\}$ where $\mathscr{C}(F)$ is as defined in (5).

As in the initial induction step, we will assign to each $F \in \mathscr{F}(n+1)$ an order which will, in a sense, classify the sets of $\mathscr{F}(n+1)$ according to how "far away" they are from 0 . But since we want to assure that $\delta_{n+1} \subset \delta_{n}$, or, what is the same thing, $L_{n+1}(x) \subset L_{n}(x)$ for all $x \in X$, we must take more care in defining the order of an element $F$ of $\mathscr{F}(n+1)$. Because each $F \in \mathscr{F}(n+1)$ is contained in an unique element of $\mathscr{F}(n)$, the "predecessors" of the elements of $F$ must be contained in the set of "predecessors" of that unique element of $\mathscr{F}(n)$ which contains it.

We will partition $\mathscr{F}(n+1)$ into the sets $\mathscr{F}(n+1, t), t=0,1, \cdots$, and if $F \in \mathscr{F}(n+1, t)$ we will say $F$ has order $t$. First we let

$$
\mathscr{F}(n+1,0)=\{F \in \mathscr{F}(n+1): 0 \in F\} \text {. }
$$

Let $F_{n}$ be an element of $\mathscr{F}(n, s-1)$ and suppose that order has been defined for the elements of some subset of $\mathscr{C}\left(F_{n}\right)$ which contains at least $\left\{F \in \mathscr{P}\left(F_{n}\right): F \cap \partial_{*} F_{n} \neq \varnothing\right\}$. Let $F$ be an element of $\mathscr{C}\left(F_{n}\right)$ such that $F \cap C^{\prime}\left(F_{n}\right) \neq \varnothing$ and such that order has not yet been defined for $F$. We will let $F$ be an element of $\mathscr{F}(n+1, t)$ and say $F$ has order $t$ if $t$ is the smallest positive integer such that there exists $F_{*} \in \mathscr{C}\left(F_{n}\right)$ such that $F_{*} \subset C\left(F_{n}\right), F_{*}$ has order $t-1$, and $F_{*} \cap F \cap$ int $F_{n} \neq \varnothing$. Let

$$
\begin{aligned}
\mathscr{B}_{*}(F)= & \left\{F_{*} \in \mathscr{C}\left(F_{n}\right): F_{*} \in \mathscr{F}(n+1, t-1), F_{*} \subset C\left(F_{n}\right)\right. \\
& \text { and } \left.F_{*} \cap F \cap \operatorname{int} F_{n} \neq \varnothing\right\} .
\end{aligned}
$$

Notice that this is enough to define order for all $F \in \mathscr{C}\left(F_{n}\right)$ such that $F \cap C^{\prime}\left(F_{n}\right) \neq \varnothing$, since $C^{\prime}\left(F_{n}\right)$ is connected and covered by the connected sets $\left[F \cap \operatorname{int} F_{n}\right] \cup\left[F \cap \partial_{*} F_{n}\right]$. Now suppose $F \in \mathscr{C}\left(F_{n}\right)$ but $F \cap C^{\prime}\left(F_{n}\right)=\varnothing$. Then $F \subset P$ for some unique $P \in \mathscr{P}\left(F_{n}\right)$, where $\mathscr{P}\left(F_{n}\right)$ is as defined in the proof of Lemma 2. Let $F$ be an element of $\mathscr{F}(n+1, t)$ and say $F$ has order $t$ if $t$ is the smallest positive integer such that there exists some $F_{*} \in \mathscr{F}(n+1, t-1)$ such that $F_{*} \subset P$ and $F \cap F_{*} \cap$ int $P \neq \varnothing$. Let

$$
\begin{aligned}
\mathscr{E}_{*}(F)= & \left\{F_{*} \in \mathscr{C}\left(F_{n}\right): F_{*} \in \mathscr{F}(n+1, t-1), F_{*} \subset P\right. \\
& \text { and } \left.F_{*} \cap F \cap \operatorname{int} P \neq \varnothing\right\} .
\end{aligned}
$$

This is enough to define order for all $F \in \mathscr{C}\left(F_{n}\right)$ since for each $P \in \mathscr{P}(F)$, int $P$ is connected and covered by the connected sets $F \cap$ int $P$ for $F \in \mathscr{C}\left(F_{n}, P\right)$ and $P \cap C^{\prime}\left(F_{n}\right) \neq \varnothing$. 
Suppose order has been defined for all $F \in \mathscr{G}\left(F_{n}\right)$ where $F_{n} \in \mathscr{F}(n, s-1)$. Let $F_{n, s}$ be an element of $\mathscr{F}(n, s)$ and let $F$ be an element of $\mathscr{G}\left(F_{n, s}\right)$ such that $F \cap \partial_{*} F_{n, s} \neq \varnothing$. We will let $F$ be an element of $\mathscr{F}(n+1, t)$ and say $F$ has order $t$ if $t$ is the smallest positive integer such that there exists $F_{*} \in \mathscr{F}(n+1, t-1)$ such that

$$
F_{*} \subset F_{* *} \in \mathscr{F}(n, s-1), F_{* *} \in \mathscr{E}_{*}\left(F_{n, s}\right)
$$

and $F \cap F_{*} \cap \partial_{*} F_{n, s} \neq \varnothing$. Let

$$
\begin{aligned}
\mathscr{E}_{*}(F)= & \left\{F_{*} \in \mathscr{F}(n+1, t-1): F_{*} \subset F_{* *} \in \mathscr{F}(n, s-1),\right. \\
& \left.F_{* *} \in \mathscr{E}_{*}\left(F_{n, s}\right), \text { and } F \cap F_{*} \cap \partial_{*} F_{n, s} \neq \varnothing\right\} .
\end{aligned}
$$

With this we have defined a unique order for each $F \in \mathscr{F}(n+1)$ and we have $\mathscr{F}(n+1)=\bigcup_{t} \mathscr{F}(n+1, t)$.

Now let $F$ be an element of $\mathscr{F}(n+1)$ and suppose $F \subset F_{n} \in \mathscr{F}(n)$. As mentioned earlier, in order to make the relation $\Delta$ a transitive order, it will be necessary that the elements of $\partial F_{n}-\left(\partial_{*} F_{n} \cup \partial^{*} F_{n}\right)$ have no successors. To ensure that this happens since $\partial_{*} F$ will have successors in the relation $\delta_{n+1}$, we must define $\delta_{*} F$ for $F \in \mathscr{C}\left(F_{n}\right)$ so that

$$
\partial_{*} F \cap\left[\partial F_{n}-\left(\partial_{*} F_{n} \cup \partial^{*} F_{n}\right)\right]=\varnothing,
$$

when $F \cap \partial_{*} F_{n}=\varnothing$. Also, if $F \cap C\left(F_{n}\right)=\varnothing$ and $F \subset P \in \mathscr{P}\left(F_{n}\right)$, we want $\partial_{*} F \cap \partial P=\varnothing$.

We do this as follows. If $F \in \mathscr{C}\left(F_{n}\right)$ and $F \cap \partial_{*} F_{n} \neq \varnothing$, set

$$
\partial_{*} F=F \cap \partial_{*} F_{n} \text {. }
$$

If $F \cap \partial_{*} F_{n}=\varnothing$, but $F \cap C^{\prime}\left(F_{n}\right) \neq \varnothing$, for each $E \in \mathscr{E}_{*}(F)$ choose $p(F, E) \in F \cap E \cap$ int $F_{n}$. Let

$$
\begin{array}{r}
T\left(F_{n}\right)=\left\{p(F, E): F \in \mathscr{C}\left(F_{n}\right), F \cap \partial_{*} F_{n}=\varnothing,\right. \\
\left.F \cap C^{\prime}\left(F_{n}\right) \neq \varnothing \text { and } E \in \mathscr{E}_{*}(F)\right\} .
\end{array}
$$

Since $T\left(F_{n}\right)$ is a finite set it is a closed subset of int $F_{n}$. Because: $F_{n}$ is normal, we can find $S\left(F_{n}\right)$, an open subset of $F_{n}$ such that

$$
C l\left(S\left(F_{n}\right)\right) \cap T\left(F_{n}\right)=\varnothing \text { and } \partial F_{n} \subset S\left(F_{n}\right) .
$$

Then for $F \in \mathscr{C}\left(F_{n}\right)$ such that $F \cap \partial_{*} F_{n}=\varnothing$ and $F \cap C^{\prime}\left(F_{n}\right) \neq \varnothing$, set.

$$
\partial_{*} F=\left[F \cap\left(\cup \mathscr{E}_{*}(F)\right)\right]-S\left(F_{n}\right) .
$$

Since $\mathscr{E}_{*}(F) \neq \varnothing$ and for $E \in \mathscr{E}_{*}(F), p(F, E) \notin S\left(F_{n}\right)$, it follows that $\partial_{*} F$ is a nonempty closed subset of $\partial F$ and $\partial_{*} F \cap\left[\partial F_{n}-\partial_{*} F_{n}\right]=\varnothing$. Similarly for $F \in \mathscr{G}\left(F_{n}\right)$ such that $F \cap C^{\prime}\left(F_{n}\right)=\varnothing$, we know that $F \subset P$ for some unique $P \in \mathscr{P}\left(F_{n}\right)$. Now for each $F \subset P$ such that $F \cap C^{\prime}\left(F_{n}\right)=\varnothing$ and each $E \in \mathscr{E}_{*}(F)$, we can choose $p(F, E)$ to be an 
element of $F \cap E \cap \operatorname{int} P$. Let

$$
T\left(F_{n}, p\right)=\left\{p(F, E): F \in \mathscr{C}\left(F_{n}, P\right), F \cap C^{\prime}\left(F_{n}\right)=\varnothing \text {, and } E \in \mathscr{E}_{*}(F)\right\} .
$$

Since $T\left(F_{n}, P\right)$ is a finite set it is a closed subset of int $P$. Therefore we can find an open set $S\left(F_{n}, P\right)$ such that $\partial P \subset S\left(F_{n}, P\right)$ and

$$
C l\left(S\left(F_{n}, P\right)\right) \cap T\left(F_{n}, P\right)=\varnothing .
$$

Now for each $F \subset P$ such that $F \cap C^{\prime}\left(F_{n}\right)=\varnothing$, set

$$
\partial_{*} F=\left[F \cap\left(\cup \mathscr{E}_{*}(F)\right)\right]-S\left(F_{n}, P\right) .
$$

It follows that $\partial_{*} F \cap \partial P=\varnothing$ and $\partial_{*} F$ is not empty.

For all $F \in \mathscr{F}(n+1)$, let

$$
\mathscr{E}^{*}(F)=\left\{E \in \mathscr{F}(n+1): F \in \mathscr{E}_{*}(E)\right\}
$$

and let

$$
\partial^{*} F=\cup\left\{\partial_{*} E \cap F: E \in \mathscr{E}^{*}(F)\right\} .
$$

If $E \in \mathscr{E}^{*}(F)$ let

$$
\partial_{E} F=\partial_{*} E \cap F
$$

Let

$$
\mathscr{f}(F)=\left\{E \in \mathscr{F}(n+1): E \neq F,(E \cap F)-\left(\partial_{*} F \cup \partial^{*} F\right) \neq \varnothing\right\}
$$

and for $E \in \mathscr{E}(F)$ let

$$
\partial_{E} F=C l\left[(E \cap F)-\left(\partial_{*} F \cup \partial^{*} F\right)\right] .
$$

If $\mathscr{f}^{*}(F) \neq \varnothing$, let $\rho_{1}(F)=d\left(\partial_{*} F, \partial^{*} F\right)$ and if $\mathscr{E}^{*}(F)=\varnothing$, let $\rho_{1}(F)=$ $\operatorname{diam} F$. If $F \cap \partial_{*} F_{n}=\varnothing$ but $F \cap C^{\prime}\left(F_{n}\right) \neq \varnothing$, let $\rho_{2}(F)=d\left(\partial_{*} F, \partial F_{n}\right)$. If $F \cap C^{\prime}\left(F_{n}\right)=\varnothing$, and $F \subset P \in \mathscr{P}\left(F_{n}\right)$, let $\rho_{2}(F)=d\left(\partial_{*} F, \partial P\right)$; otherwise let $\rho_{2}(F)=\operatorname{diam} F$. Finally let

$$
\rho(F)=\min \left\{\rho_{1}(F), \rho_{2}(F)\right\} .
$$

This completes the definitions necessary to define $\delta_{n}$ for all positive integers $n$.

We now define a relation $\Delta$ on $X$ by letting $\Delta=\bigcap_{i=1}^{\infty} \delta_{i}$. It remains to show that $\Delta$ is a partial order satisfying Theorem 2 .

3. The relation $\Delta$ satisfies the hypotheses of Koch's Arc Theorem. The relation $\Delta$ is continuous on $X$ since $\Delta=\bigcap_{n=1}^{\infty} \delta_{n}$ and we have shown in (7) that each $\delta_{n}$ is closed in $X \times X$. Also 0 is a zero for $\Delta$ since $0 \in L(x)$ for all $x \in X$. We must further show that $L(x)$, the set of predecessors of each $x \in X$ under the relation $\Delta$, is a 
connected set. To do this it is enough to show that $L_{n+1}(x) \subseteq L_{n}(x)$ for each $x \in X$, since then the set $\left\{L_{n}(x)\right\}_{n=1}^{\infty}$ will be a nest of continua and $L(x)=\bigcap_{n=1}^{\infty} L_{n}(x)$ will be a continuum and thus be connected.

Because $L_{n+1}(x),(6)$, is a union of sets of the forms $J(F)$ and $J(F, E)$ where $F \in \mathscr{F}(n+1), E \in \mathscr{E}(F) \cup \mathscr{E}^{*}(F)$ and $x \in C(F)$ or $x \in C(F, E)$ to prove $L_{n+1}(x) \subset L_{n}(x)$, it is sufficient to show that if $x \in F \in \mathscr{F}(n+1)$ and $F \subset F^{\prime} \in \mathscr{F}(n)$, then $F \cup J(F)$ is a subset of either $J\left(F^{\prime}\right)$ or $J\left(F^{\prime}, E^{\prime}\right)$ for some $E^{\prime} \in \mathscr{E}\left(F^{\prime}\right) \cup \mathscr{E}^{*}\left(F^{\prime}\right)$. This proof is omitted but is a straightforward induction on $t$ when

$$
x \in F \subset F^{\prime} \in \mathscr{F}(n, t)
$$

using definitions (2.1-2) of $J(F)$ and (3.1-4) of $\mathscr{E}_{*}(F)$.

It is clear that $\Delta$ is reflexive. That $\Delta$ is a partial order on $X$ will be established by the following lemmas.

Lemma 3. Let $F_{1}$ and $F_{2}$ be distinct elements of $\mathscr{F}(n)$ and let $x$ be an element of $\partial F_{1}-\left(\partial_{*} F_{1} \cup \partial^{*} F_{1}\right)$. Then $x$ is an element of $\partial F_{2}-$ $\left(\partial_{*} F_{2} \cup \partial^{*} F_{2}\right)$.

Proof. We will proceed by induction on $n$. Suppose $n=1$, and that $F_{1}$ is an element of $\mathscr{F}(1, t)$. Then the order of $F_{2}$ is either $t-1$, $t$, or $t+1$, using (1) since $F_{1} \cap F_{2} \neq \varnothing$. If $F_{2} \in \mathscr{F}(1, t-1)$ then by (4.1) $x \in \partial_{*} F_{1}$ and if $F_{2} \in \mathscr{F}(1, t+1)$ by (4.1) $x \in \partial^{*} F_{1}$, and both of these situations contradict the hypothesis. Thus $F_{2} \in \mathscr{F}(1, t)$. Suppose $x \in \partial_{*} F_{2}$. Then there exists a set $F_{3} \in \mathscr{F}(1, t-1)$ such that $x \in F_{2} \cap F_{3}$. But also $x \in F_{1}$ so $x \in F_{1} \cap F_{3} \subset \partial_{*} F_{1}$ which is a contradiction. Similarly, if $x \in \partial^{*} F_{2}$ there exists a set $F_{3} \in \mathscr{F}(1, t+1)$ such that $x \in F_{3} \cap F_{2}$, so $x \in F_{1} \cap F_{3} \subset \partial^{*} F_{1}$ and we get another contradiction.

We now suppose the lemma is true for $n=1,2, \cdots, k-1$. Let $F_{1}$ and $F_{2}$ be distinct elements of $\mathscr{F}(k)$ and suppose $F_{1} \subset T_{1} \in \mathscr{F}(k-1)$ and $F_{2} \subset T_{2} \in \mathscr{F}(k-1)$. By $(4.1-4)$ we have for $i=1,2$

$$
\partial_{*} F_{i} \cup \partial^{*} F_{i} \subset\left(\operatorname{int} T_{i}\right) \cup \partial_{*} T_{i} \cup \partial^{*} T_{i} .
$$

So $x \notin \partial_{*} F_{1} \cup \partial^{*} F_{1}$ implies by (4.2) that $x \notin \partial_{*} T_{1} \cup \partial^{*} T_{1}$.

Now if $T_{1} \neq T_{2}, x \in T_{1} \cap T_{2}$ implies $x \in \partial T_{1} \cap \partial T_{2}$. From the induction hypothesis $x \in \partial T_{2}-\left(\partial_{*} T_{2} \cup \partial^{*} T_{2}\right)$. Therefore by $(10) x \in \partial F_{2}-\left(\partial_{*} F_{2} \cup \partial^{*} F_{2}\right)$.

If, however, both $F_{1}$ and $F_{2}$ are subsets of $T_{1}$, we will consider first the case when $x \in C\left(T_{1}\right)$. If $x \in S\left(T_{1}\right)$, where $S\left(T_{1}\right)$ is as defined in (8) then by (4.3) $x \notin \partial_{*} F \cup \partial^{*} F$ for any $F \in \mathscr{G}\left(T_{1}\right)$ such that $F \cap C\left(T_{1}\right) \neq \varnothing$. In particular $x \notin \partial_{*} F_{2} \cup \partial^{*} F_{2}$. If $x \notin S\left(T_{1}\right)$, the argument that $x \notin \partial_{*} F_{2} \cup \partial^{*} F_{2}$ is analogous to the situation when $n=1$.

The final case when $x \notin C\left(T_{1}\right)$ follows by a similar argument using that either $F_{1} \subset P_{1}$ and $F_{2} \subset P_{2}$ where $P_{1} \neq P_{2}$ and $P_{1}$ and $P_{2}$ are in 
$\mathscr{P}\left(T_{1}\right)$; or $F_{1} \cup F_{2} \subset P$ for some $P \in \mathscr{P}\left(T_{1}\right)$ and that

$$
\partial_{*} F_{i} \cup \partial^{*} F_{i} \subset\left[P-S\left(T_{1}, P\right)\right] \cup \partial^{*} T_{1}
$$

for $i=1,2$.

Note. It follows from Lemma 3 that for $x \in\left(\right.$ int $\left.F_{1}\right) \cup \partial_{*} F_{1} \cup \partial^{*} F_{1}$ where $F_{1} \in \mathscr{F}(n, t)$ and if $x \in F_{2}$ for some $F_{2} \in \mathscr{F}(n), F_{2} \neq F_{1}$, then $x \in \partial_{*} F_{2} \cup \partial^{*} F_{2}$. Further if $x \in \partial_{*} F_{1}$ then $F_{2} \in \mathscr{F}(n, t-1) \cup \mathscr{F}(n, t)$, and if $x \in \partial^{*} F_{1}$, then $F_{2} \in \mathscr{F}(n, t) \cup \mathscr{F}(n, t+1)$.

Lemma 4. Let $F \in \mathscr{F}(n)$ and $x \in \partial F-\left(\partial_{*} F \cup \partial^{*} F\right)$. Let $m$ be an integer such that there exists $E \in \mathscr{F}(m)$ such that $x \in E \subset F$ and $E \cap\left(\partial_{*} F \cup \partial^{*} F\right)=\varnothing$. Then $C\left(E, E^{*}\right) \cap \partial F=\varnothing$ for all $E^{*} \in \mathscr{E}^{*}(E)$.

Proof. Let $F_{m}=E$ and $F_{n}=F$. Then there exists $\left\{F_{i}\right\}_{i=n}^{m}$ such that $F_{i} \in \mathscr{F}$ (i) and $F_{i+1} \subset F_{\imath}$. Let $l$ be the greatest integer such that $m>l \geqq n$ and $\partial_{*} E-\partial_{*} F_{l} \neq \varnothing$ and let $k$ be the greatest integer such that $\partial^{*} E-\partial^{*} F_{k} \neq \varnothing$. Then $m>l \geqq n$ and $m>k \geqq n$. Without loss of generality suppose $l \geqq k$. Since $\partial_{*} E \subset \partial_{*} F_{l+1}$,

$$
d\left(\partial_{*} E, \partial F_{l}\right) \geqq d\left(\partial_{*} F_{l+1}, \partial F_{l}\right)
$$

From (8) $\partial F_{l} \subset S\left(F_{l}\right)$ and by (4.3) and (4.4) $\partial_{*} F_{l+1} \subset F_{l} \backslash S\left(F_{l}\right)$. Therefore

$$
d\left(\partial_{*} F_{l+1}, \partial F_{l}\right)=\rho_{2}\left(F_{l}\right) \geqq \rho\left(F_{l}\right) \text { by }(9) \text {. }
$$

Thus $d\left(\partial_{*} E, \partial F_{l}\right) \geqq \rho\left(F_{l}\right)$. Similarly

$$
d\left(\partial_{*} E, \partial F_{k}\right) \geqq d\left(\partial^{*} F_{k+1}, \partial F_{k}\right) \geqq \rho\left(F_{k}\right) .
$$

Also

$$
d\left(\partial^{*} E, \partial F\right) \geqq d\left(\partial^{*} E, \partial F_{k}\right) \quad \text { and } \quad d\left(\partial_{*} E, \partial F\right) \geqq d\left(\partial_{*} E, \partial F_{l}\right) .
$$

Thus

$$
\begin{aligned}
d\left(\partial^{*} E \cup \partial^{*} E, \partial F\right) & \geqq \min \left\{d\left(\partial^{*} E, \partial F_{k}\right), d\left(\partial_{*} E, \partial F_{l}\right)\right\} \\
& >\min \left\{\rho\left(F_{k}\right), \rho\left(F_{l}\right)\right\}=\rho\left(F_{l}\right) \geqq \rho\left(F_{m-1}\right) .
\end{aligned}
$$

From Lemma 2 if $x \in \partial E$ and

$$
d\left(x, \partial_{*} E \cup \partial^{*} E\right)>\frac{\rho\left(F_{m-1}\right)}{3}
$$

then $x \in C\left(E, E^{*}\right)$ for any $E^{*} \in \mathscr{E}^{*}(E)$. Thus $\partial F \cap C\left(E, E^{*}\right)=\varnothing$ for any $E^{*} \in \mathscr{C}^{*}(E)$.

Lemma 5. Let $x \in \partial F-\left(\partial^{*} F \cup \partial_{*} F\right)$ for $F \in \mathscr{F}(n)$. Then $x$ has no successors other than itself in the relation $\Delta$. 
Proof. Assume $y \geqq_{\Delta} x$ and $y \neq x$. Choose $m>n$ such that $d(x, y)>2^{-m}$ and such that $d\left(x, \partial_{*} F \cup \partial^{*} F\right)>2^{-m}$. Then since for $F^{\prime} \in \mathscr{F}(m)$ we have $\operatorname{diam} F^{\prime}<2^{-m}, x$ and $y$ are not both elements of any one $F^{\prime} \in \mathscr{F}(m)$. Also, if

$$
x \in F^{\prime} \in \mathscr{F}(m) \text {, then } F^{\prime} \cap\left(\partial_{*} F \cup \partial^{*} F\right)=\varnothing,
$$

so that $m$ satisfies the conditions of Lemma 5. However, since $x \in L_{m}(y)$ and $x$ is in no element of $\mathscr{F}(m)$ containing $y$, by $(6) x \in C\left(F^{\prime}, F^{*}\right)$ for some

$$
F^{\prime} \in \mathscr{F}(m) \text { and } F^{*} \in \mathscr{E}^{*}\left(F^{\prime}\right) \text {. }
$$

But by Lemma $4, C\left(F^{\prime}, F^{*}\right) \cap \partial F=\varnothing$. This is a contradiction and proves that such a $y$ cannot exist.

In the next lemma we will use the following notation. If $x \in$ int $F$ for some $F \in \mathscr{F}(n, t)$, set $q_{n}(x)=t$. If $x \in \partial_{*} F$ for some $F \in \mathscr{F}(n, t)$, set $q_{n}(x)=t-1$. By the note after Lemma $3, q_{n}(x)$ is well-defined and single valued for all $x \in(\operatorname{int} F) \cup \partial_{*} F \cup \partial^{*} F$ where $F \in \mathscr{F}(n)$.

\section{LEMMA 6. The relation $\Delta$ is anti-symmetric.}

Proof. Assume there exist $x$ and $y$ in $X$ such that $x \neq y, x \leqq_{\triangle} y$ and $y \leqq_{4} x$. Choose $n$ such that $d(x, y)>2^{-n+1}$. Then, since $x \in L_{n}(y)$, there exists some $F_{1} \in \mathscr{F}(n)$ such that $y \in F_{1} \in \mathscr{F}(n, t)$ and $x \in J\left(F_{1}\right)$. By Lemma $5, y \in\left(\operatorname{int} F_{1}\right) \cup \partial_{*} F_{1} \cup \partial^{*} F_{1}$, so $q_{n}(y)$ is defined and $q_{n}(y) \geqq$ $t-1$. Now because $d(x, y)>2^{-n+1}, x \in F_{2} \in \mathscr{F}(n)$ where $F_{2} \in \mathscr{F}(n, s)$ and $s<t-1$. Also by Lemma $5, x \in$ int $F_{2} \cup \partial_{*} F_{2} \cup \partial^{*} F_{2}$, so $q_{n}(x)$ is defined and $q_{n}(x) \leqq s<t-1$. It follows that $q_{n}(y)>q_{n}(x)$. But by a symmetric argument since $y \in L_{n}(x)$, it can be shown that $q_{n}(x)>$ $q_{n}(y)$. This contradiction proves that $\Delta$ is anti-symmetric.

Lemma 7. The relation $\Delta$ is transitive.

Proof. Let $x, y$ and $z$ be elements of $X$ such that $x \leqq_{\triangle} y$ and $y \leqq_{\triangleleft} z$. We will show $x \leqq_{\lrcorner} z$. We can assume $x<y$ and $y<z$. Choose $n$ such that $\min \{d(x, y), d(y, z), d(x, z)\}>2^{-n+1}$. It is enough to show $x \in L_{n}(z)$ since we have shown $L_{n-1}(z) \supset L_{n}(z)$. Since $y \in L_{n}(z), y \in F_{y}$ for some $F_{y} \in \mathscr{F}(n, t)$ where $y \in J\left(F_{y}, E^{\prime}\right) \subset L_{n}(z)$ for some $E^{\prime} \in \mathscr{C}^{*}\left(F_{y}\right)$. By Lemma 4, $y \in \operatorname{int} F_{y} \cup \partial_{*} F_{y} \cup \partial^{*} F_{y}$. If $y \in$ int $F_{y}$ then since

$$
x \in L_{n}(y), x \in J\left(F_{y}\right) \subset J\left(F_{y}, E^{\prime}\right) \subset L_{n}(z) .
$$

If $y \notin$ int $F_{y}$ then either $y \in \partial_{*} F_{y}$ or $y \in \partial^{*} F_{y}$. We will consider the case when $y \in \partial_{*} F_{y}$. The argument is similar when $y \in \partial^{*} F_{y}$. By the note after Lemma 3 if $y \in F \in \mathscr{F}(n)$, then $F \in \mathscr{F}(n, t) \cup \mathscr{F}(n, t-1)$. 
If $y \in F_{*} \in \mathscr{F}(n, t-1)$ where $x \in J\left(F_{*}\right)$ then $x \in J\left(F_{*}, F_{y}\right) \subset L_{n}(z)$. If we assume this is not the case then $x \notin J\left(F_{*}, F_{y}\right)$ for any $F_{*} \in \mathscr{E}_{*}\left(F_{y}\right)$. Let $\mathscr{A}=\left\{F_{*} \in \mathscr{F}(n, t-1): x \in J\left(F_{*}, F\right)\right.$ for some $F \in \mathscr{F}(n, t)$ such that $y \in F\}$. The set $\mathscr{A}$ is not empty since $x \in L_{n}(y)$. Let

$$
r=\min \left\{d\left(y, F_{*}\right): F_{*} \in \mathscr{A}\right\} \text {. }
$$

Since $y \notin F_{*}$ for any $F_{*} \in \mathscr{A}, r>0$. Choose $m>n$ such that $r>2^{-m}$. Now because $x \in L_{m}(y) \subset L_{n}(y)$ there exists a set $T \in \mathscr{F}(m)$ such that $y \in T$ and $x \in J(T)$. Either $T \subset F$ for some $F \in \mathscr{F}(n, t)$ or $T \subset F_{*}$ for some $F_{*} \in \mathscr{F}(n, t-1)$. However if

$$
T \subset F_{*} \in \mathscr{F}(n, t-1), x \in J(T) \subset J\left(F_{*}, F_{y}\right)
$$

which contradicts our assumption. Thus there exists $F \in \mathscr{F}(n, t)$ such that $T \subset F$. Now by $(2.2) x \in J(T) \subset T \cup \cup\left\{J\left(T_{*}, T\right): T_{*} \cap T \neq \varnothing\right.$, $T_{*} \in \mathscr{E}_{*}(T)$ and $T_{*} \subset F_{*}$ for some $\left.F_{*} \in \mathscr{F}(n, t-1)\right\}$. By the choice of $m$ and $r, F_{*} \notin \mathscr{L}$. But $x \in J(T) \subset J\left(F_{*}, F\right)$ implies that $F_{*} \in \mathscr{A}$. This contradiction says that $x \in J\left(F_{*}, F_{y}\right)$ for some $F_{*} \in \mathscr{E}_{*}\left(F_{y}\right)$ and thus $x \in J\left(F_{y}\right) \subset L_{n}(z)$. This completes the proof that $\Delta$ is transitive.

\section{REFERENCES}

1. R. H. Bing, Partitioning a set, Bull. Amer. Math. Soc. 55 (1949), 1101-1110.

2. R. J. Koch, Arcs in partially ordered spaces, Pacific J. Math. 9 (1959), 723-728.

3. R. L. Moore, On the foundations of plane analysis situs, Trans. Amer. Math. Soc. 17 (1966), 131-164.

4. L. E. Ward, Jr., Concerning Koch's theorem on the existence of arcs, Pacific J. Math. 15 (1965), 347-355.

Received December 9, 1968.

UNIVERSITY OF VICTORIA

VICTORIA, B. C.

Willa METTE UNIVERSITY

Salem, Oregon 



\section{PACIFIC JOURNAL OF MATHEMATICS}

\section{EDITORS}

H. ROYDEN

Stanford University

Stanford, California

Richard Pierce

University of Washington

Seattle, Washington 98105
J. DugundJI

Department of Mathematics

University of Southern California

Los Angeles, California 90007

BASIL GORDON

University of California

Los Angeles, California 90024

\section{ASSOCIATE EDITORS}

E. F. BECKENBACH

B. H. NeUmanN

F. WOLF

K. YOSHIDA

\section{SUPPORTING INSTITUTIONS}

UNIVERSITY OF BRITISH COLUMBIA

CALIFORNIA INSTITUTE OF TECHNOLOGY

UNIVERSITY OF CALIFORNIA

MONTANA STATE UNIVERSITY

UNIVERSITY OF NEVADA

NEW MEXICO STATE UNIVERSITY

OREGON STATE UNIVERSITY

UNIVERSITY OF OREGON

OSAKA UNIVERSITY

UNIVERSITY OF SOUTHERN CALIFORNIA
STANFORD UNIVERSITY

UNIVERSITY OF TOKYO

UNIVERSITY OF UTAH

WASHINGTON STATE UNIVERSITY

UNIVERSITY OF WASHINGTON

$* * \quad * \quad *$
AMERICAN MATHEMATICAL SOCIETY
CHEVRON RESEARCH CORPORATION
TRW SYSTEMS
NAVAL WEAPONS CENTER




\section{Pacific Journal of Mathematics}

Vol. 30, No. $1 \quad$ September, 1969

William Wells Adams, Simultaneous diophantine approximations and cubic irrationals ..................................... 1

Heinz Bauer and Herbert Stanley Bear, Jr., The part metric in convex

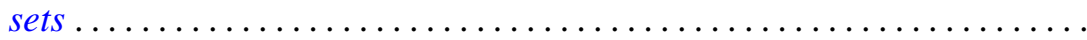

L. Carlitz, A note on exponential sums ...................... 35

Vasily Cateforis, On regular self-injective rings ................. 39

Franz Harpain and Maurice Sion, A representation theorem for measures on infinite dimensional spaces ......................... 47

Richard Earl Hodel, Sum theorems for topological spaces .............. 59

Carl Groos Jockusch, Jr. and Thomas Graham McLaughlin, Countable

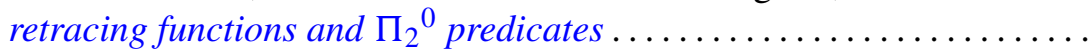

Bjarni Jónsson and George Stephen Monk, Representations of primary

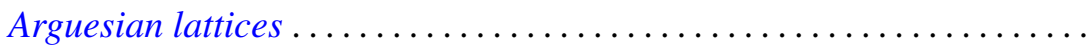

Virginia E. Walsh Knight, A continuous partial order for Peano continua...................................... 141

Kjeld Laursen, Ideal structure in generalized group algebras ........... 155

G. S. Monk, Desargues' law and the representation of primary lattices . . . 175

Hussain Sayid Nur, Singular perturbation of linear partial differential equation with constant coefficients ..........................

Richard Paul Osborne and J. L. Stern, Covering manifolds with cells ... 201

Keith Lowell Phillips and Mitchell Herbert Taibleson, Singular integrals in several variables over a local field...

James Reaves Smith, Local domains with topologically $T$-nilpotent radical....

Donald Platte Squier, Elliptic differential equations with discontinuous coefficients .................................

Tae-il Suh, Algebras formed by the Zorn vector matrix...

Earl J. Taft, Ideals in admissible algebras . .................... 259

Jun Tomiyama, On the tensor products of von Neumann algebras........ 263

David Bertram Wales, Uniqueness of the graph of a rank three group ..... 271

Charles Robert Warner and Robert James Whitley, A characterization of regular maximal ideals ......................... 